\title{
MIXING ALGORITHMS FOR FIXED-POINT ITERATIONS IN SELF-CONSISTENT ELECTRONIC STRUCTURE CALCULATIONS
}

\author{
M. Novák*, R. Cimrman ${ }^{* *}$, V. Lukeš ${ }^{* * *}$, E. Rohan ${ }^{\dagger}$, J. Vackáŕr ${ }^{\ddagger}$
}

\begin{abstract}
In ab-initio calculations of electronic structure and material properties within the density-functional theory (DFT) framework, a self-consistent stationary state of a many-electron system is sought by a fixedpoint iteration of Kohn-Sham equations, the so called DFT loop. One of the key components needed for fast convergence is to apply a suitable mixing of new and previous states in the DFT loop. We discuss performance of the standard Anderson/Pulay class mixing algorithms as well as a newly proposed adaptable hybrid scheme that combines those approaches so as to accelerate the convergence. The scheme is used within our computer implementation of a new robust ab-initio real-space code based on (i) density functional theory, (ii) finite element method and (iii) environment-reflecting pseudopotentials.
\end{abstract}

Keywords: fixed-point mixing, finite element method, electronic structure, density functional theory

\section{Introduction}

The electronic structure of a material determines its chemical/physical properties, such as elasticity, hardness, electric and magnetic properties, etc., see (Martin, 2005). The electronic structure calculations represent a rigorous tool for predicting and understanding the properties of materials for many years, and a number of methods and algorithms has been proposed and implemented in various computer codes. Many of those methods are based on the density-functional theory (DFT) framework (Martin, 2005), and seeking of a self-consistent state by a fixed-point iteration, the so called DFT loop. One of the key components needed for fast convergence is to apply a suitable mixing of new and previous states in the DFT loop as it can potentially both accelerate the convergence and reduce oscillations in the self-consistence error.

We have developed a new robust ab-initio real-space code based on (i) the density functional theory, (ii) the finite element method (FEM) (Strang and Fix, 2008) and (iii) environment-reflecting pseudopotentials (Vackár and Šimůnek, 2003). This approach to solving Kohn-Sham equations and calculating electronic states, total energy, Hellmann-Feynman forces and material properties brings a new quality particularly for non-crystalline, non-periodic structures (Vackár et al., 2011). The convergence properties of the code related to the used discretization (a standard finite element basis, or a spline basis of isogeometric analysis) were analyzed in papers (Cimrman et al., 2018b,a).

In this contribution we discuss convergence of the code with the standard Anderson mixing algorithm ((Anderson, 1965; Pulay, 1982), called also Pulay mixing) and its modification Guaranteed Residual Pulay method ((Bowler and Gillan, 2000), abbreviated as GR-Pulay) as well as a newly proposed adaptable hybrid scheme that combines those two approaches so as to accelerate the convergence. The presented numerical examples illustrate behaviour of the schemes on a simple system (Nitrogen molecule) and a more complex system (a graphene fragment), where all the schemes struggle.

\footnotetext{
Matyáš Novák, Department of Mechanics, Faculty of Applied Sciences, University of West Bohemia \& Institute of Physics, Academy of Sciences of the Czech Republic, novakmat@fzu.cz

** Robert Cimrman, New Technologies - Research Centre, University of West Bohemia, cimrman3@ntc.zcu.cz

*** Vladimír Lukeš, Department of Mechanics, Faculty of Applied Sciences, University of West Bohemia, vlukes@kme.zcu.cz

$\dagger$ Eduard Rohan, Department of Mechanics, Faculty of Applied Sciences, University of West Bohemia, rohan@kme.zcu.cz

‡ Jirí Vackář, Institute of Physics, Academy of Sciences of the Czech Republic, vackar@ fzu.cz
} 


\section{Electronic structure calculations and FEM}

The systems of atoms and molecules are described in the most general form by the many-particle Schrödinger equation, which is, however, too complicated to solve, even for three electrons. Among the techniques reducing this complexity, we use the DFT approach (Dreizler and Gross, 1990). The DFT allows decomposing the many-particle Schrödinger equation into the one-electron Kohn-Sham equations (Kohn and Sham, 1965), that have, after the FE discretization, the following eigenvalue problem form, seeking the orbitals $\psi_{i}$ :

$\int_{\Omega} \frac{1}{2} \nabla \psi_{i} \cdot \nabla v \mathrm{~d} V+\int_{\Omega} v V \psi_{i} \mathrm{~d} V=\varepsilon_{i} \int_{\Omega} v \psi_{i} \mathrm{~d} V, \quad \forall v \in H_{0}^{1}(\Omega)$

where $V:=V_{\mathrm{H}}+V_{\mathrm{xc}}+\hat{V}$ is the total potential consisting of an effective ionic potential for electrons $\hat{V}$, the exchange-correlation potential $V_{\mathrm{xc}}$ describing the non-coulomb electron-electron interactions and the electrostatic potential $V_{\mathrm{H}}$ obtained as a solution to the Poisson equation

$$
\int_{\Omega} \nabla v \cdot \nabla V_{\mathrm{H}}=4 \pi \int_{\Omega} \rho v, \quad \forall v \in H_{0}^{1}(\Omega)
$$

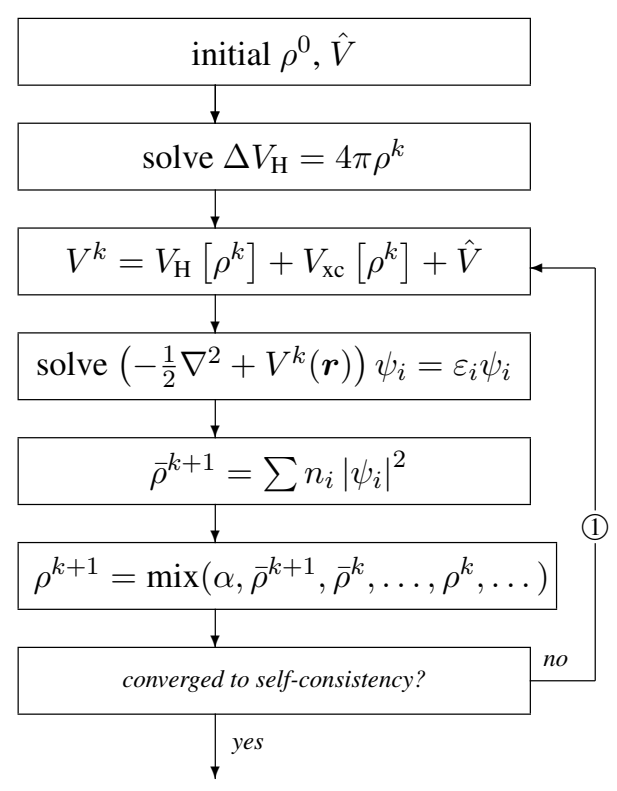

Fig. 1: The DFT loop.

with the charge density $\rho(\boldsymbol{r})=\sum_{i}^{N} n_{i}\left|\psi_{i}(\boldsymbol{r})\right|^{2}$ on the right-hand side. See (Cimrman et al., 2018b) for details. The problem (1), (2) is nonlinear and needs to be solved iteratively: we use the stadard DFT loop algorithm outlined in Fig. 1. The purpose of the DFT loop is to find a self-consistent solution - a fixed point of a function of the charge density $\rho$. For this task, a variety of nonlinear solvers can be used. Below we use several quasi-Newton solvers applied to $\operatorname{DFT}\left(\rho^{k}\right)-\rho^{k}=\rho^{k+1}-\rho^{k}=0$, where DFT denotes a single iteration of the DFT loop.

\section{Examples}

The two test systems are a nitrogen molecule $\mathrm{N}_{2}$ as an easy to solve system, with only 10 eigenpairs required, and a graphene fragment as an example of a more complex system, with 84 eigenpairs required. For illustration, the resulting charge densities are shown in Fig. 2. The standard Anderson (Anderson,
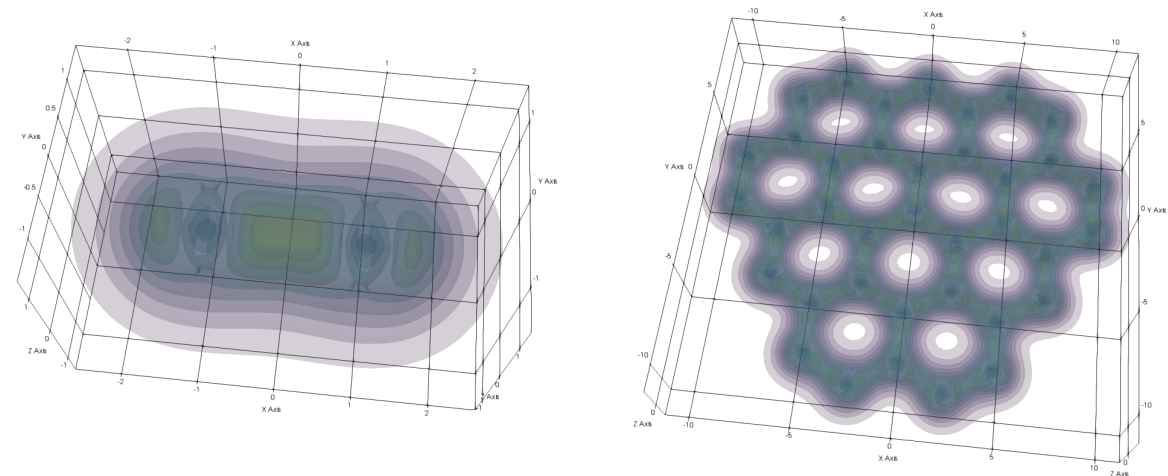

Fig. 2: Charge densities $\rho$ of the test systems: left: a $N_{2}$ molecule, right: a graphene fragment.

1965; Pulay, 1982) and GR-Pulay (Bowler and Gillan, 2000) mixing algorithms were used. As the GRPulay algorithm failed to converge in the graphene fragment case, we proposed a modification, abbreviated as GR-Pulay-LM: the new charge density was generated using the standard linear mixing scheme, instead of the fixed $\alpha=1.0$ of the original GR-Pulay. This is similar to, but not the same as, the approach in (Banerjee et al., 2016). The last algorithm was our newly proposed adaptable hybrid scheme. The convergence graphs for various values of the mixing parameter $\alpha$ are shown in Fig. 3 for the $\mathrm{N}_{2}$ molecule, and in Fig. 4 for the graphene fragment. As the criteria for stopping iterations, we used either a stagnation 
of convergence (no residual reduction in ten subsequent iterations) or the residual norm decrease below a threshold.
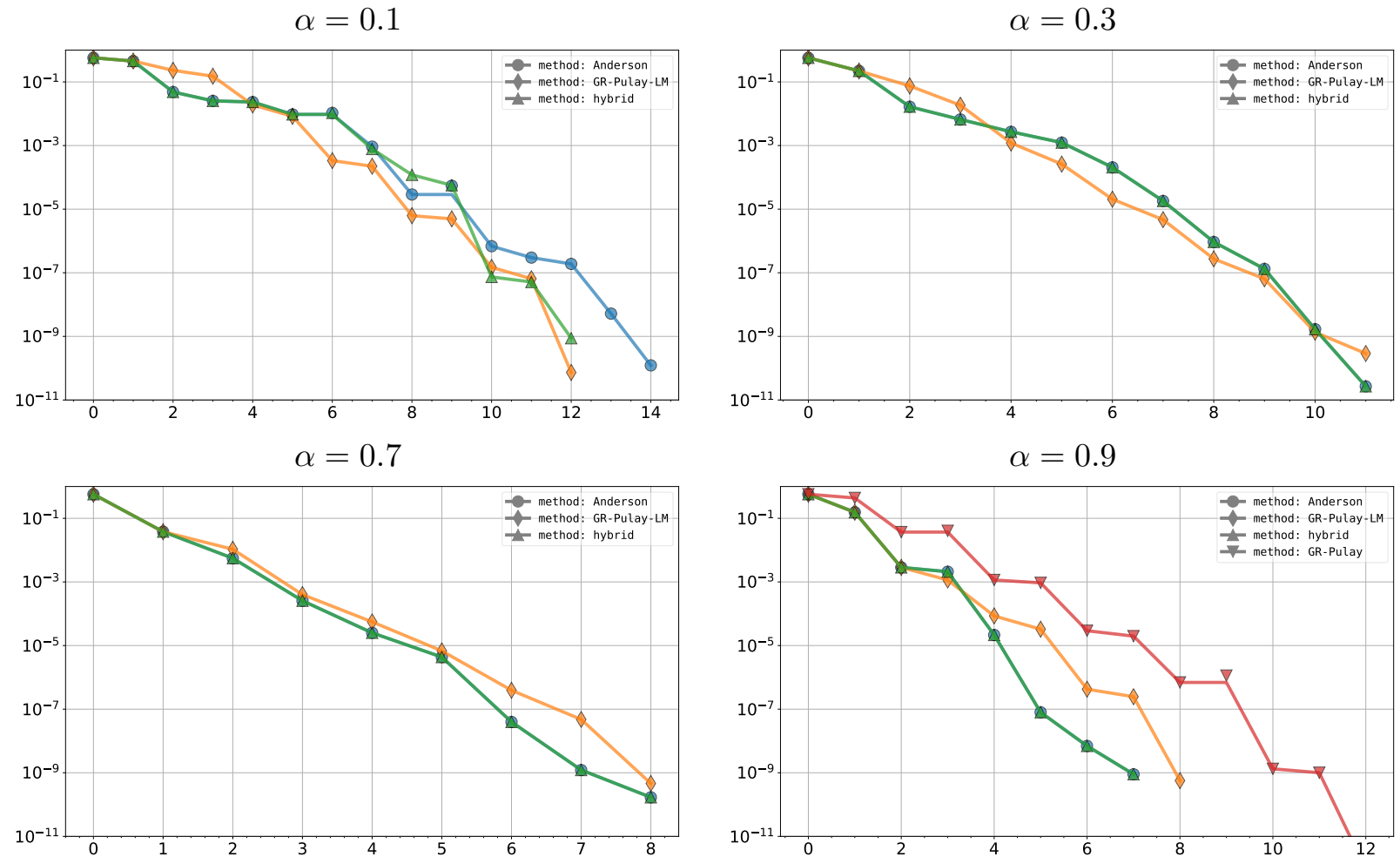

Fig. 3: Convergence of the DFT loop for different values of the mixing parameter $\alpha$ in the case of the $\mathrm{N}_{2}$ molecule. The original GR-Pulay algorithm $(\alpha=1)$ is added to the $\alpha=0.9$ plot for comparison.
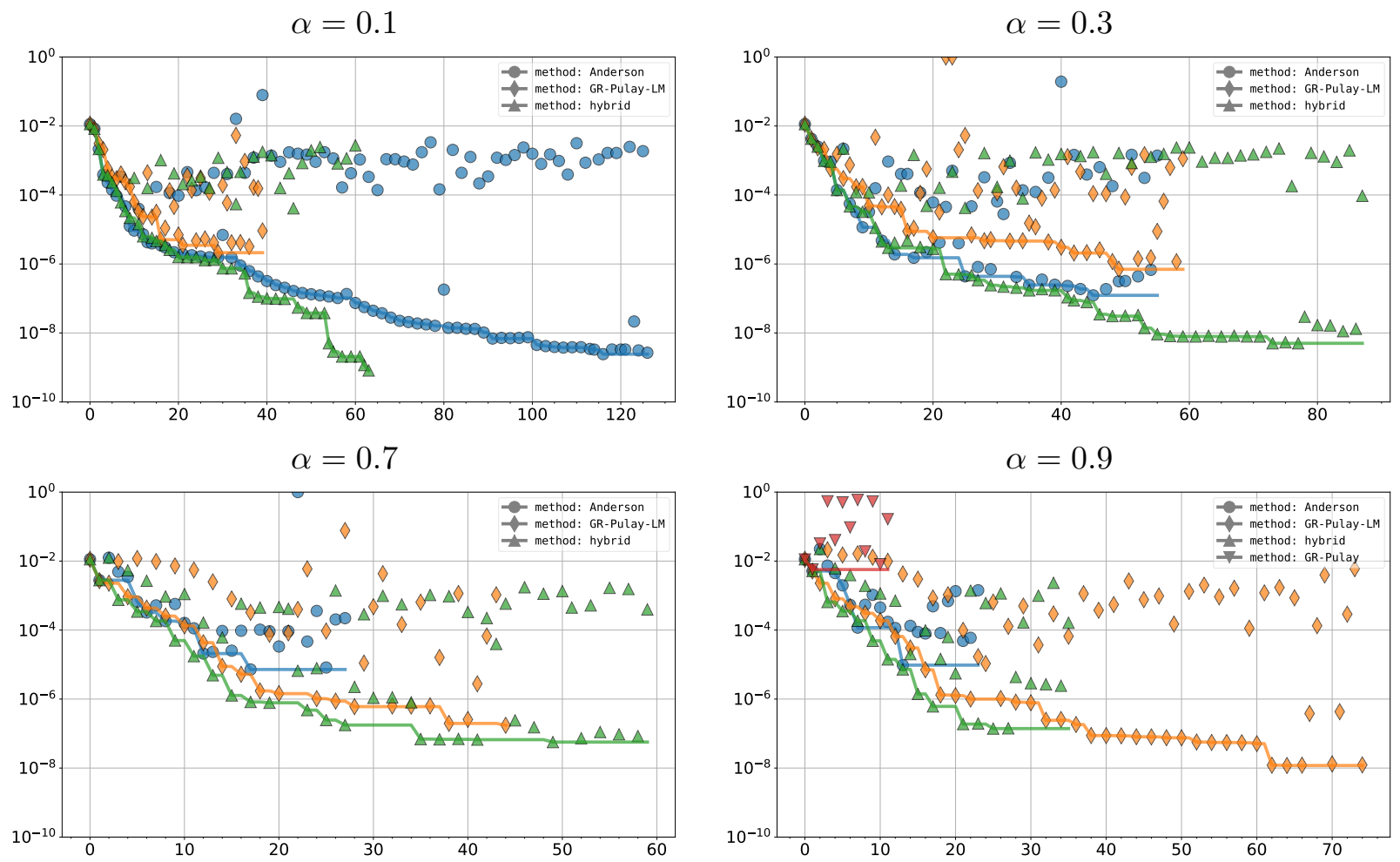

Fig. 4: Convergence of the DFT loop for different values of the mixing parameter $\alpha$ in the case of the graphene fragment. The solid lines connect the running minima of the oscillating convergence curves. The original GR-Pulay algorithm $(\alpha=1)$ is added to the $\alpha=0.9$ plot for comparison. 


\section{Conclusion}

The proposed adaptable hybrid scheme performs better or as well as the best other scheme in both test problems, independently on the value of the mixing parameter. The GR-Pulay-LM scheme (our modification of the GR-Pulay algorithm) is competitive especially for the higher mixing parameter values in the complex test problem. In future we plan to compare the properties of the proposed scheme to other recently published schemes such as (Banerjee et al., 2016).

\section{Acknowledgments}

The work was supported by the Czech Science Foundation, grant project GA17-12925S. The first author acknowledges the support by CEDAMNF project, reg. no. CZ. 02.1.01/0.0/0.0/15_003/0000358, co-funded by the ERDF as part of the Ministry of Education, Youth and Sports OP RDE programme.

\section{References}

Anderson, D. G. (1965). Iterative procedures for nonlinear integral equations. Journal of the ACM (JACM), 12(4):547560.

Banerjee, A. S., Suryanarayana, P., and Pask, J. E. (2016). Periodic pulay method for robust and efficient convergence acceleration of self-consistent field iterations. Chemical Physics Letters, 647:31 - 35.

Bowler, D. and Gillan, M. (2000). An efficient and robust technique for achieving self consistency in electronic structure calculations. Chemical Physics Letters, 325(4):473-476.

Cimrman, R., Novák, M., Kolman, R., Tůma, M., Plešek, J., and Vackář, J. (2018a). Convergence study of isogeometric analysis based on bézier extraction in electronic structure calculations. Applied Mathematics and Computation, 319:138 - 152.

Cimrman, R., Novák, M., Kolman, R., Tůma, M., and Vackář, J. (2018b). Isogeometric analysis in electronic structure calculations. Mathematics and Computers in Simulation, 145:125 - 135.

Dreizler, R. M. and Gross, E. K. U. (1990). Density Functional Theory. Springer-Verlag.

Kohn, W. and Sham, L. J. (1965). Self-consistent equations including exchange and correlation effects. Phys. Rev., 140(4A):A1133-A1138. doi:10.1103/PhysRev.140.A1133.

Martin, R. M. (2005). Electronic Structure: Basic Theory and Practical Methods. Cambridge University Press.

Pulay, P. (1982). Improved scf convergence acceleration. J. Comput. Chem., 3:55 6-560.

Strang, G. and Fix, G. (2008). An Analysis of the Finite Element Method. Wellesley-Cambridge Press, Wellesley. pp. 414.

Vackár̆, J. and Šimůnek, A. (2003). Adaptability and accuracy of all-electron pseudopotentials. Phys. Rev. B, 67. 125113.

Vackář, J., Čertík, O., Cimrman, R., Novák, M., Šipr, O., and Plešek, J. (2011). Advances in the Theory of Quantum Systems in Chemistry and Physics, volume 22 of Prog. Theoretical Chem. and Phys., chapter Finite Element Method in Density Functional Theory Electronic Structure Calculations, pages 199-217. Springer. 